\title{
The exacerbation of cutaneous psoriasis induced by anti - TNF therapy - case report
}

\author{
Anca Costache ${ }^{1}$, Mădălina Ionela Chiriac ${ }^{2}$, Elena Rezuş ${ }^{\star, 1,2}$ \\ ${ }^{1}$ Rheumatology Department, Rehabilitation Hospital lasi, ${ }^{2}$ "Grigore T. Popa" University of Medicine \\ and Pharmacy lasi, Romania
}

\begin{abstract}
The tumor necrosis factor (TNF) blockers are highly effective therapy for numerous autoimmune inflammatory diseases, including rheumatoid arthritis, psoriatic arthropathy (PsA), spondyloarthropathies, juvenile idiopathic arthritis and inflammatory bowel disease. Paradoxically, these agents can induce cutaneous and systemic autoimmune diseases; the most common are psoriasis skin lesions and systemic lupus erythematosus (SLE) induced by TNF blockers. We present the case of a 59 years-old female, diagnosed at 32 years with cutaneous psoriasis vulgaris and psoriatic arthropathy at 54 years-old. After treatment failure with conventional Disease-Modifying Antirheumatic Drugs (DMARDs), biologic therapy with infliximab was started. After 3 years of treatment, the patient suffers an exacerbation of skin lesions and worsening joint damage. We decided to stop the infliximab and initiate the therapy with another anti-TNF agent (adalimumab), with improvement of the skin lesions and joint manifestations.
\end{abstract}

Keywords: psoriasis, biologic therapy, psoriatic arthropathy, adalimumab

\section{Introduction}

In the last years, TNF antagonists such as infliximab (IFX), adalimumab (ADA), etanercept, golimumab, certolizumab have revolutionized the treatment of autoimmune inflammatory diseases and were authorized for the treatment of cutaneous psoriasis. The target for anti-TNF agents in patients with psoriasis is to reduce the inflammatory cytokines leading to a decrease in the cutaneous lesions of neutrophils, $\mathrm{T}$ cells and dendritic cells and epidermal hyperplasia.

Received: April 2014; Accepted after review: May 2014; Published: June 2014

${ }^{*}$ Corresponding author: Elena Rezuş, Rheumatology Department, Rehabilitation Hospital, lasi, "Grigore T. Popa" University of Medicine and Pharmacy, lasi, Romania. Email: elena rezus@yahoo.com
Cutaneous adverse effects of TNF blockers include infections, eczema and allergic skin reactions. Several cases of vasculitis, SLE and cutaneous malignancies are registered [1, 2].

There are reports of several cases of cutaneous psoriasis that occurred during therapy with anti-TNF agents and cases of exacerbation of skin lesions [1]. Some patients may have severe skin manifestations requiring interruption of biological therapy, which can lead to worsening of the underlying disease.

\section{Case report}

In February 2012, a 59 year-old female was admitted to Rheumatology Clinic accusing inflammatory polyarthralgias at small joints of hands: metacarpal-phalange (MCP), proximal 
interphalangeal (PIP) and distal interphalangeal (DIP), wrists, right elbow, coxofemoral joints, knees and ankles bilaterally accompanied with morning stiffness about an hour and erythemato-squamous generalized skin lesions. The patient had been diagnosed with psoriatic arthropathy about 5 years before and the diagnosis was based on Classification Criteria for Psoriatic Arthritis (CASPAR). The past history of illness describes that the patient was diagnosed with cutaneous psoriasis vulgaris since the age of 32 (22 years before the occurrence of joint lesions), and then nail psoriasis (onycholysis, hyperkeratosis), $3^{\text {rd }}$ grade arterial hypertension, $3^{\text {rd }}$ grade obesity, type 2 diabetes mellitus, dyslipidemic syndrome, secondary bilateral osteoarthritis of the hip and knee. After being diagnosed with PsA, the patient received methotrexate (MTX), an initial dose of $15 \mathrm{mg}$ per week (oral administration) with the improvement of joint damage as well as the skin lesions for approximately 1 year.

Subsequently, the patient presented an active form of PsA and we decided to increase the dose of MTX to $20 \mathrm{mg} /$ week (subcutaneously). After 3 months with increased dose of MTX, the patient was still presenting the active form of PsA, therefore leflunomide $20 \mathrm{mg}$ per day (oral administration) was added to the medical treatment. Despite the association of the two DMARDs, the patient continued to present tenderness (tender joints count- TJC $=28$ ) and swelling (swollen joints count- $\mathrm{SJC}=18$ ) on a large number of joints and Psoriasis Area and Severity Index (PASI) score $=30$, which indicates a significant skin damage.

The latent tuberculosis infection was excluded (proven by normal appearance of the chest radiography and negative QuantiFERON test), as well as screening for viral hepatitis (negative HBsAg and anti HCV antibodies). We decided to initiate the biologic therapy with IFX (in March 2009) at a dose of $5 \mathrm{mg} / \mathrm{kg}$ body weight after standard therapeutic protocol (at 2, 6 and 8 weeks later). The clinical outcome after 3 months indicated a significant improvement of the skin lesions (PASI score = 4), as well as a decrease of TJC, SJC and disease activity score. Conventional synthetic DMARDs therapy (MTX and leflunomide) was continued until March 2011, when the treatment was stopped because of hepatocytolysis.

In June 2011, the hepatocytolysis and cholestasis syndrome persisted (with elevated ALT and AST, 4 times than normal value); in consequence, IFX monotherapy was continued with an increase of dose to $10 \mathrm{mg}$ $/ \mathrm{kg}$ body weight parenteral at 8 weeks. The present clinical examination reveals the erythemato-squamous lesions disposed in patches and plaques on the scalp, retroauricular, on chest, abdomen, upper and lower limbs (Fig. 1, 2).

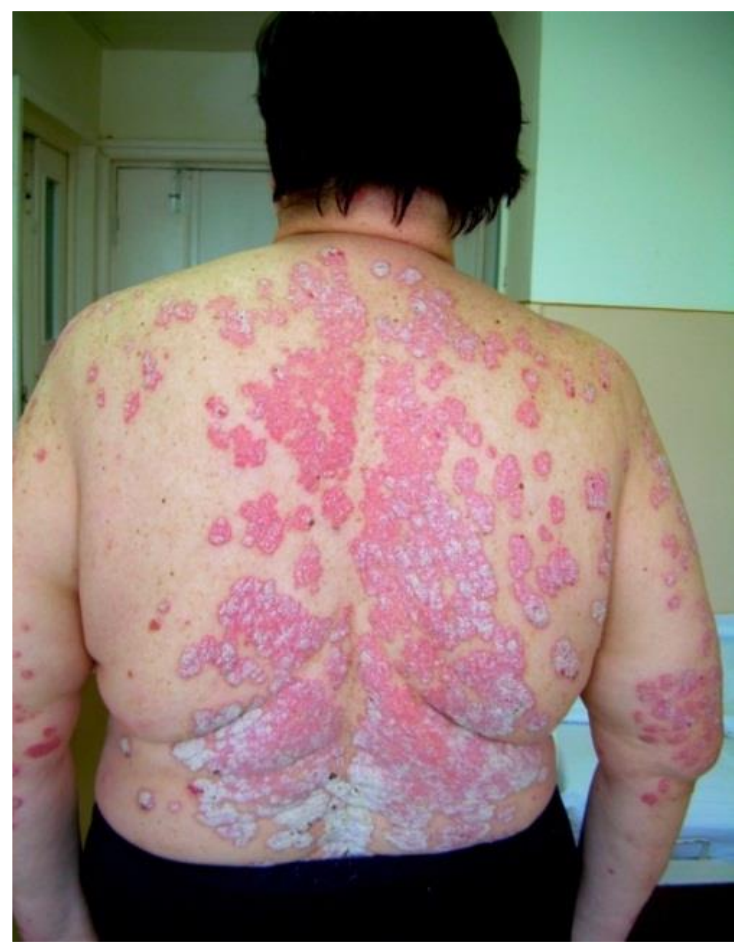

Fig. 1. Erythematous -squamous lesions, patches and confluent plaques on the posterior chest, right and left upper limbs

Also, onycholysis and hyperkeratosis were identified. Musculoskeletal examination showed difficulty in walking, with walking cane support, dorsal kyphosis and lumbar paravertebral muscle contracture with pain on palpation of the spinous processes, and lumbar mobility limitation (Shober test $=10+1$ $\mathrm{cm})$. 


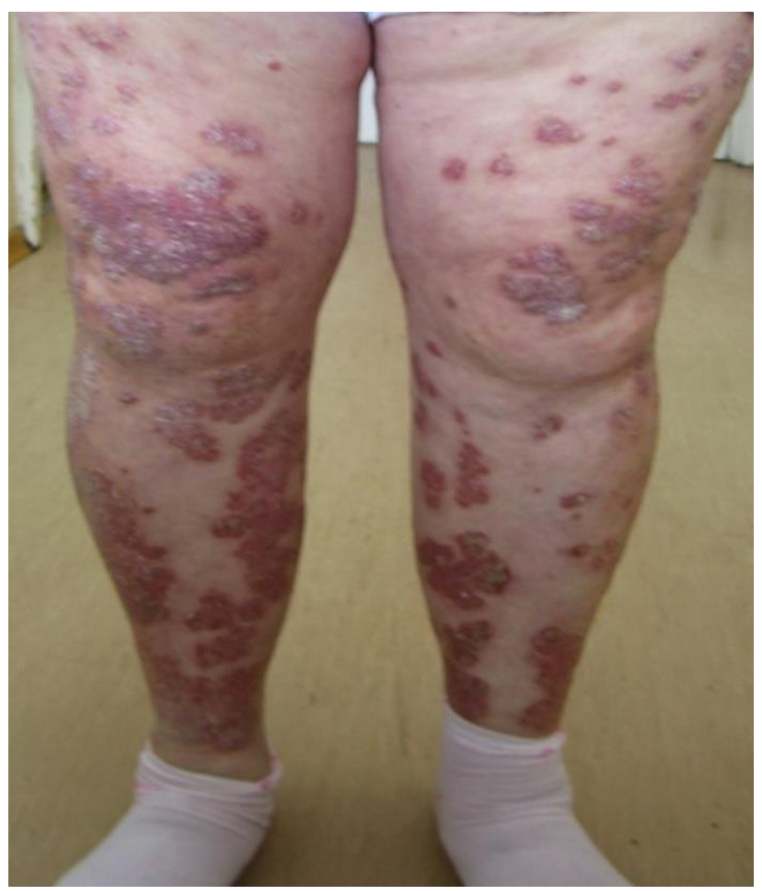

Fig. 2. Erythematous -squamous lesions, patches and plaques of lower limbs

The patient presented painful limitation of motion range at the peripheral joints: shoulders, elbows, wrists and small joints of the hands (MCP, PIP, and DIP), knee, ankle joints, metatarsal, a total of 22 tender joints on palpation. Ten out of these joints were swollen.

Laboratory findings: inflammatory syndrome $(E S R=35 \mathrm{~mm} / 1 \mathrm{~h}$ and $C R P=2.4$ $\mathrm{mg} / \mathrm{l}$ ), normocytic normochromic anemia (HGB $=11.2 \mathrm{mg} / \mathrm{dl}$ ), normal liver and kidney functional tests, and mild hyperuricemia (7mg/dl).

In conclusion, clinical examination and laboratory tests revealed an active form of PsA (disease activity score on DAS $28=7.4$ indicating a highly active form of disease) and extensive skin lesions (score calculated PASI= 25). Therefore we consider this as corresponding to an exacerbation of skin lesions induced by biological therapy with IFX.

The patient was examined by a dermatologist, who decided to initiate the topical corticosteroids therapy. Therapy with IFX was suspended and replaced by another anti-TNF agent, ADA $40 \mathrm{mg}$ subcutaneously, every other week. The clinical outcome at 3 months after initiation of therapy with ADA revealed the improvement of skin lesions evidenced by PASI score $=4.5$, significant improvement of PsA with a decreased number of painful and swollen joints (tender joints count $=10$, swollen joints count $=2$ ), DAS $28=$ 4.7 (medium disease activity), and the absence of biological inflammatory syndrome.

\section{Discussions}

Systematic retrospective studies reported that the new onset or exacerbation of psoriasis is a class effect of anti-TNF agents, several similar cases being reported in the literature, even for newer anti-TNF agents like as golimumab [1]. No correlations to age or sex were reported in different studies on diseases treated with anti-TNF agents such as skin psoriasis, rheumatoid arthritis, ankylosing spondylitis and other spondyloarthropathies (including psoriatic arthropathy), Behcet's disease, inflammatory bowel disease and juvenile idiopathic arthritis [1]. In contrast with psoriasis induced by other drugs, the skin biopsies taken from psoriasiform lesions induced by treatment with anti-TNF agents identified similar histological changes as those found in idiopathic psoriasis [2]. It was noticed that patients with spondyloarthropathies frequently develop pustular lesions [2]. The mechanism underlying this phenomenon is still unclear. The most commonly accepted hypothesis is the discrepancy between the interferon-alpha (IFN- $\alpha$ ) and TNF- $\alpha$ [3]. The plasmacytoid dendritic cells induce psoriasis through IFN- $\alpha$ production, infiltrating the skin in early phase of psoriasis [2]. Commonly, the plasmacytoid cells are inhibited by TNF- $\alpha$ which impedes the hematopoietic precursor maturation. The inhibition of TNF- $\alpha$ with monoclonal antibodies causes the increase of IFN- $\alpha$ production and therefore promotes or exacerbates the psoriasis $[4,5]$. There are several studies that support this hypothesis: the increased levels of MxA protein (an intracellular marker of IFN signaling pathways) in cells of inflammatory cutaneous lesions induced by anti-TNF agents. Furthermore, cases of de novo psoriasis or exacerbation of psoriasis after IFN- $\alpha$ administration or topical 
application of imiquimod (a potent inducer of IFN- $\alpha$ ) were reported [6].

The period between the first administration of anti-TNF agents and development or psoriasis exacerbation is extremely variable, suggesting the involvement of an external trigger factor. The frequencies of this adverse effect among patients that receive anti-TNF agents also suggest the involvement of genetic factors [5].

There is no consensus on therapeutic management of such cases. Patients should be evaluated for possible viral or bacterial infection through blood cultures, urine culture, stool cultures or other biological products. Moreover, it is necessary to identify various trigger factors of cutaneous rash: post-trauma stress, including psycho-emotional stress. All patients should consult a dermatologist for evaluation and possible skin biopsy, in particular for those requiring differential diagnosis with other skin diseases. The therapeutic approach depends on the extension and tolerability of skin lesions. In patients with severe skin lesions or who don't want to continue the treatment, anti-TNF therapy should be suspended and replaced by administration of more aggressive local therapy (corticosteroids, keratolytic agents, vitamin $D$ analogues) under the supervision of a dermatologist. In patients with tolerable skin lesions, it is recommended to use another antiTNF agent. In patients with skin lesions that are not amenable, the therapy with MTX,

\section{References}

1. Ko JM, Gottlieb AB, Kerbleski JF: Induction and exacerbation of psoriasis with TNF-blockade therapy: a review and analysis of 127 cases. $J$ Dermatolog Treat, 2009, 20(2):100-8.

2. Joyau C, Veyrac G, Dixneuf V, Jolliet P: Antitumour necrosis factor alpha therapy and increased risk of de novo psoriasis: is it really a paradoxical side effect? Clin Exp Rheumatol, 2012, 30(5):700-6.

3. Takahashi $\mathrm{H}$, Hashimoto $\mathrm{Y}$, Ishida-Yamamoto $A$, et al: Psoriasiform and pustular eruption induced by infliximab. J Dermatol, 2007, 34:468-72. acitretin or cyclosporine under the supervision of a dermatologist is recommended $[7,8]$.

\section{Conclusions}

The tumor necrosis factor (TNF) blockers are highly effective therapy for numerous autoimmune inflammatory diseases. However, these agents can cause cutaneous and systemic autoimmune diseases, the most common being psoriasiform skin lesions and systemic lupus erytematosus (SLE) induced by TNF blockers.

The pathogenic mechanism remains actually unknown, but there is the hypothesis of a difference between the interferon $\alpha$, he TNF and plasmacytoid cell proliferation. These make the difficulty of therapeutic approach; in severe cases, it was indicated to suspend the anti-TNF agents and initiate the local aggressive treatment; in other cases, substitution to another anti-TNF agent was preferred. In refractory cases, therapy with MTX, acitretin or cyclosporine may be recommended.

In our case, after treatment failure with conventional DMARDs, biologic therapy with infliximab was instituted and after 3 years of treatment, the patient suffers an exacerbation of skin lesions and worsening joint damage. Infliximab treatment was stopped and therapy with adalimumab was initiated, with certain improvement of the skin lesions and joint manifestations.

4. Nestle FO, Conrad C, Tun-Kyi A, et al: Plasmacytoid predendritic cells initiate psoriasis through interferon-production. J Exp Med, 2005, 202:135-43.

5. Eriksen KW, Lovato P, Skov L, et al: Increased sensitivity to interferon- in psoriatic T cells. $J$ Invest Dermatol, 2005, 125:936-44.

6. Gilliet M, Conrad C, Geiges M, et al: Psoriasis triggered by toll-like receptor 7 agonist imiquimod in the presence of dermal plasmacytoid dendritic cell precursors. Arch Dermatol, 2004, 140:1490-5. 
7. Goiriz R, Dauden E, Perez-Gala S, Guhl G, Garcia-Diez A: Flare and change of psoriasis morphology during the course of treatment with tumour necrosis factor blockers. Clin Exp Dermatol, 2007, 32:176-9.
8. Collamer A N, Battafarano DL: Psoriatic Skin Lesions Induced by Tumor Necrosis Factor Antagonist Therapy: A Literature Review and Potential Mechanisms of Action. Arthritis \& Rheumatism (Arthritis Care \& Research), 2008, 59(7):996-1001. 